\title{
EXPERIENCE OF INTRODUCTION OF THE TECHNOLOGY OF RECONDITIONING MICROPLASMA POWDER SURFACING AT REPAIR OF HIGH-PRESSURE TURBINE BLADES IN BATCH PRODUCTION
}

\author{
P.D. ZHEMANYUK, I.A. PETRIK and S.L. CHIGILEJCHIK \\ Company «MOTOR SICH»
}

15 Motorostroitelej Ave., 69068, Zaporozhie, Ukraine. E-mail: tb.ugmet@motorsich.com

\begin{abstract}
Features of the technology of batch repair of blades for high pressure turbines of aviation engines applied at company «MOTOR SICH», equipment, technique and technology of microplasma surfacing, surfacing consumables and heat treatment are considered. The main types of reconditioned parts are presented, and an example of metal microstructure in repair zone is given. 7 Ref., 1 Table, 7 Figures.
\end{abstract}

$\boldsymbol{K} \boldsymbol{e} \boldsymbol{y} \boldsymbol{w} \boldsymbol{o} \boldsymbol{r} \boldsymbol{s}:$ high-temperature nickel alloys, service life, thermal fatigue cracks, microplasma powder surfacing, technology

Since 1970s repair of blades of high-pressure turbines (HPT) in MOTOR SICH (reconditioning of worn edges and side walls of flanges of shroud platforms) has been performed by argon-arc surfacing process [1]. Note that our enterprise has been a pioneer in the USSR in repair of aviation blades from high-temperature nickel alloys, which at that time were regarded as absolutely unweldable. For a long time, this technology allowed performing batch-repair of such parts for a number of gas turbine engines [2].

In connection with increase of working temperature and service life on aviation engines, which were introduced comparatively recently (D18T, D436, AI222, AI450), HPT blades for operation at temperatures above $1000{ }^{\circ} \mathrm{C}$ began to be manufactured from higher alloyed hightemperature nickel alloys such as JS32-VI, JS26VI [3-5]. At operating time of more than $6,000 \mathrm{~h}$, thermal fatigue cracks of up to $6 \mathrm{~mm}$ depth were found on blades sent in for repair, in addition to operating wear of tips and side walls of shroud platforms. Argon-arc surfacing technology and consumable materials, available for this process, do not provide heat resistance properties of reconditioned surface, required for blade operation. Greater wear and, hence, deposited metal mass increased the hot cracking susceptibility at reconditioning by argon-arc process of these blades from modern complex-alloyed high-tem-

(C) P.D. ZHEMANYUK, I.A. PETRIK and S.L. CHIGILEJCHIK, 2015 perature nickel alloys (with more than $60 \% \gamma^{\prime}$ phase content).

The objective of this work is description of the features of development of surfacing technology, which allows increasing the repairability and extending the service life of HPT blades (with operating time of more than 6,000 h) from high-temperature nickel alloys JS32-VI and JS26-VI.

The main goals set by repair production for such a technology, were as follows:

- reconditioning of damaged blade sections after operation (shroud platforms, labyrinth seal edges, Z-shaped profiles and airfoil edges) on high-temperature alloys JS32-VI, JS26-VI, JS6K-VI and JS6U-VI, using filler material equivalent to base metal;

- improvement of surfacing technology through application of a constricted-arc source with precision adjustment of welding current and filler feed mechanization, that would enable surfacing performance with limited penetration depth, and mixing of deposited metal with base metal, respectively.

At that moment, PWI and SE «Ivchenko Progress» had experience of successfully solving this task. Special equipment was developed, and technology of microplasma powder surfacing (MPS) was introduced into production for reconditioning HPT blades from JS32-VI alloy for D18T engine [6]. Figure 1 shows the MPS block diagram. Deposition material in powder form is supplied by carrier gas into microplasma arc column, formed in argon atmosphere by plasma-forming nozzle channel. Heating in arc column, the powder is transferred into the weld pool, formed un- 


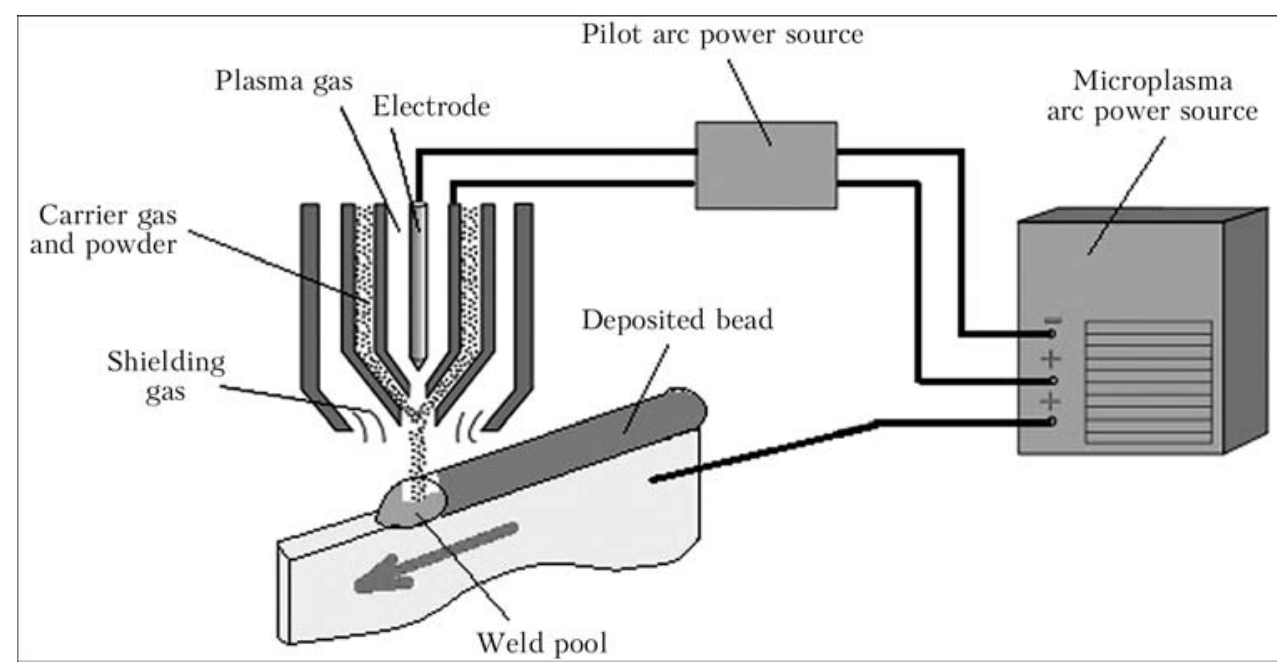

Figure 1. Schematic of MPS process

der the impact of heat flow of microplasma arc on the item (blade edge in our case). At that time, however, this process had a whole number of technological drawbacks, preventing MPS batch application at MOTOR SICH:

- broad deposited bead (more than $5 \mathrm{~mm}$ ) that greatly increased the labour consumption for machining after surfacing and the hazard of grinding cracks;

- insufficient accuracy of adjustment of surfacing modes during blade reconditioning, particularly at up to 15 A currents;

- limited applicability - surfacing of just one layer; at deposition of two or more layers increased cracking susceptibility was manifested, both during surfacing, and during subsequent heat treatment;

- large overall dimensions of equipment, particularly, plasmatron, that impaired working conditions.

Allowing for available at PWI and SE «Ivchenko Progress» experience, both positive and negative, our enterprise experts developed statement of work and on its basis Deloro Stellite (currently Kennametal Stellite, Germany) de-

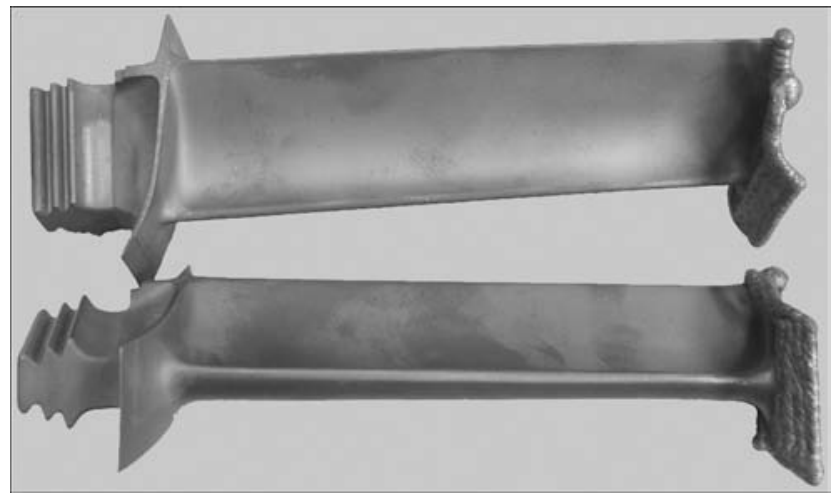

Figure 2. Blade after reconditioning of shroud platform (base metal is JS26-VI alloy, surfacing material is JS32 alloy) signed and manufactured specialized system STARWELD 190H for MPS [7], which included:

- plasmatron HPH 80;

- pilot arc power source INV 50;

- main arc power source INV 190;

- gas feeding module GT-R5-20 (carrier and shielding gas);

- plasma gas feeding module GT-S 5;

- water-cooling module LT-01;

- powder feeder PR-S2;

- control module SIEMENS Somatic S 7-300.

A package of experimental and research work was performed, which resulted in development of a complex technology of repair of HPT blades from high-temperature nickel alloys JS32-VI and JS26-VI with the following sequence of process operations.

- Surface scraping to metallic luster (removal of scale, oxides and thermal barrier coatings) from edge being surfaced to the depth of 5$10 \mathrm{~mm}$.

- Vacuum degassing annealing $(T=1250 \pm$ $\pm 10{ }^{\circ} \mathrm{C}$, soaking time of $20-30 \mathrm{~min}$, medium is vacuum of $1 \cdot 10^{-3}-1 \cdot 10^{-5} \mathrm{mbar}$ ).

- Penetrant inspection LYuM1-OV. Detected cracks are removed completely by machining.

- Building blade airfoil edge (see Figure 1) by MPS with powder from JS32-VI alloy (fraction of $+63--163 \mu \mathrm{m})$ in $1-5$ layers in STARWELD $190 \mathrm{H}$ system.

- Vacuum annealing $\left(T=960 \pm 10{ }^{\circ} \mathrm{C}\right.$, soaking time of $180-240 \mathrm{~min}$, medium is vacuum of $1 \cdot 10^{-3}-1 \cdot 10^{-5} \mathrm{mbar}$ ).

- Machining of deposited surfaces to required geometrical dimensions.

- Penetrant inspection LYuM1-OV.

- Coating restoration.

- Penetrant inspection LYuM1-OV.

Table 1 gives the main MPS parameters used in HPT blade reconditioning. 
Main technological parameters of microplasma powder surfacing

\begin{tabular}{||l|c||}
\hline \multicolumn{1}{|c|}{ Mode parameter } & Value range \\
\hline Welding current (for plasmatron HPH 80), A & $3-30$ \\
\hline Voltage, V & $20-25$ \\
\hline Plasma gas consumption, 1/min & $0.5-2.0$ \\
\hline Shielding gas consumption, 1/min & $5-15$ \\
\hline Plasma nozzle channel diameter, mm & $1.6-3.2$ \\
\hline Tungsten electrode diameter, mm & 2.4 \\
\hline Powder feed efficiency, g/min & $0.5-5.0$ \\
\hline
\end{tabular}

Effective heat input into base metal within $50-650 \mathrm{~W}$ is regulated by energy and thermal characteristics of microplasma arc. Used for this process are powders of nickel and cobalt alloys of $+63--160 \mu \mathrm{m}$ fraction, produced by the

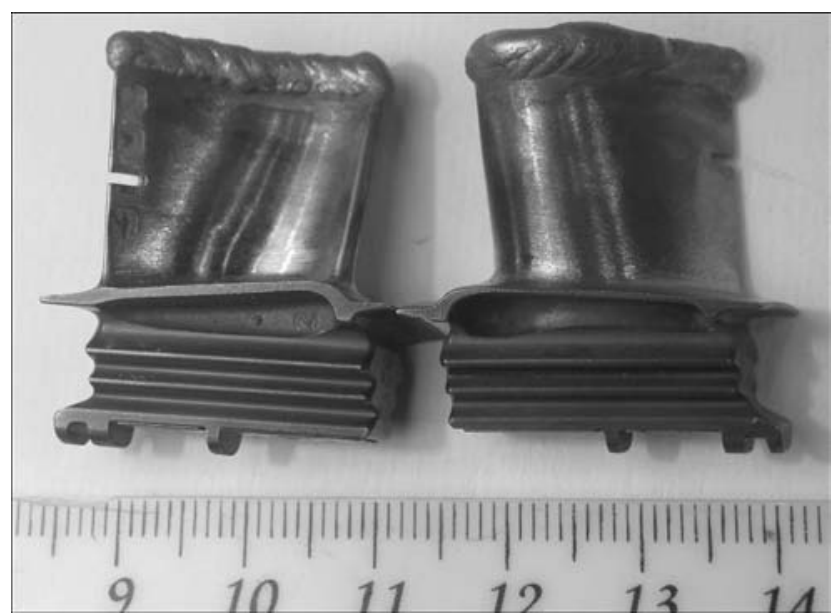

Figure 3. Blade after reconditioning of airfoil edge (base and deposited metal is JS32 alloy)

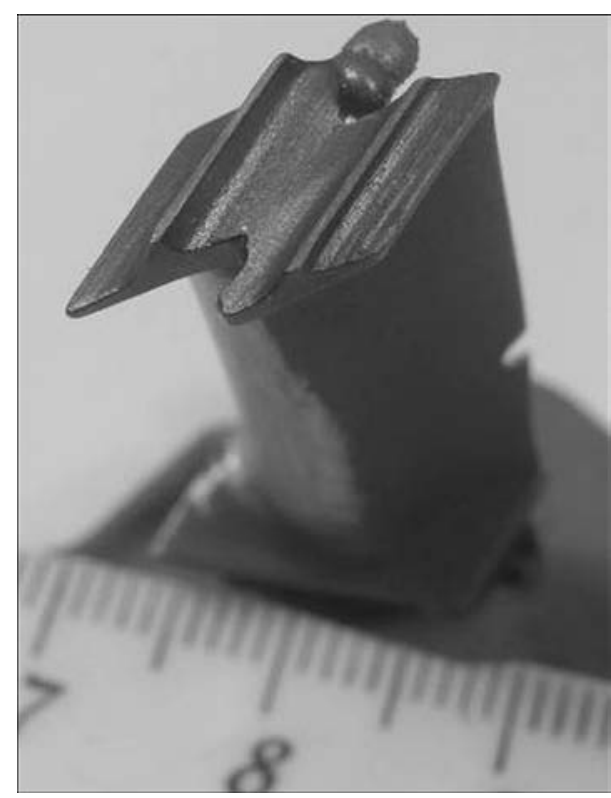

Figure 4. Blade after restoration of Z-shaped profile (base and deposited metal is JS6K alloy)

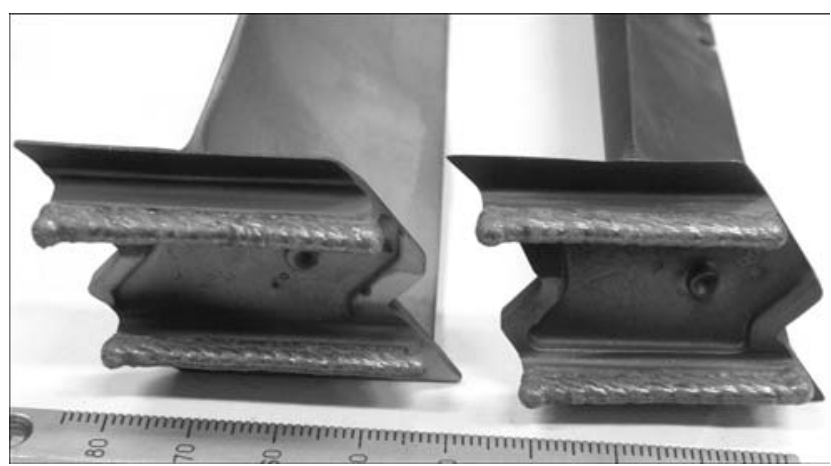

Figure 5. Blades after reconditioning labyrinth seal edges (base metal is JS6K alloy, surfacing material is Stellite 12)

method of dry atomizing of the ingot in argon atmosphere. The main used powders are from JS32-VI, JS6K-VI, V3K, Stellite 12 and Nistelle $\mathrm{C}$ alloys. Appearance of blades batch-reconditioned by MPS process is given in Figures 2-5.

Example of metallographic testing of reconditioned JS32-VI blade is given in Figures 6 and 7. Analysis of longitudinal and transverse sections showed that at 2-layer surfacing of airfoil edge by powder from JS32-VI alloy deposited layer height was equal to about $4 \mathrm{~mm}$, and its thickness was 3.0-3.5 mm (see Figure 6). HAZ was small, structural changes were equal to about $0.1-0.2 \mathrm{~mm}$ (see Figure 7). No inadmissible defects of metallurgical nature were found in the deposited metal and HAZ.

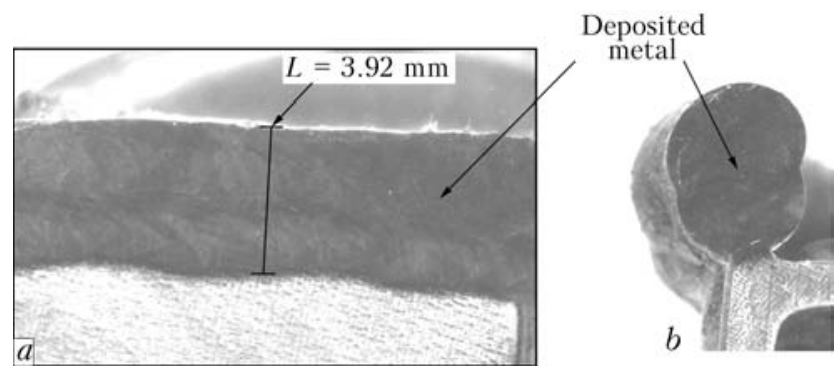

Figure 6. Appearance of longitudinal $(a)$ and transverse (b) section of reconditioned seal element of the blade

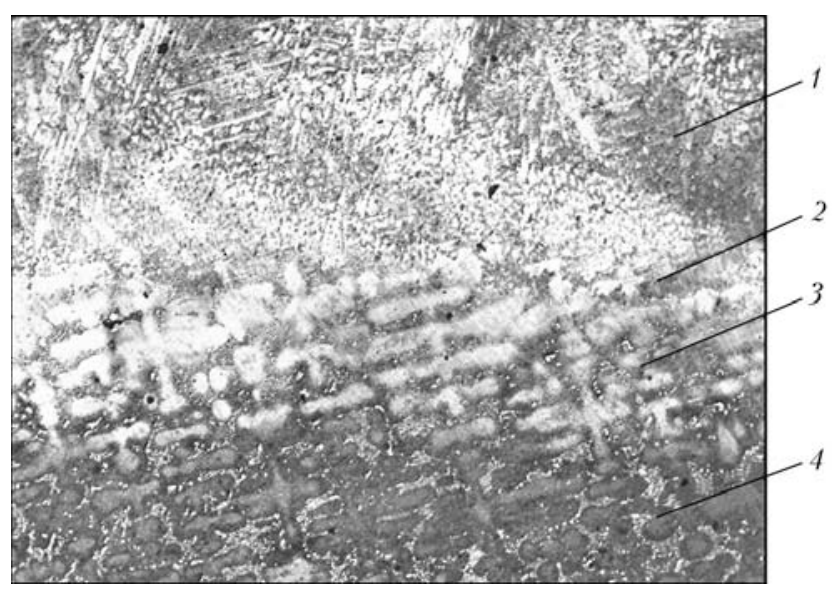

Figure 7. Microstructure $(\times 100)$ of reconditioned seal element: deposited metal JS32 (1), fusion line (2), HAZ (3) and base metal JS32-VI (4) 


\section{Conclusions}

MPS introduction at MOTOR SICH allowed improving the repairability of a considerable part of HPT blades with more than $6,000 \mathrm{~h}$ operating time. This was made possible due to mastering the technology of base metal reconditioning (surfacing in several layers) at considerable damage after operation of shroud platforms, labyrinth seal edges, Z-shaped profiles and airfoil edges from high-temperature alloys JS32-VI, JS26-VI, JS6K-VI and JS6U-VI with predominant application of equivalent filler material.

Technology of MPS of blade edges was improved through application of focused microplasma arc, precise adjustment of welding current, mechanization of disperse filler material feed and rational selection of the modes of preand postweld heat treatment.
1. Peremilovsky, I.A., Gejchenko, V.S., Frumin, I.I. (1976) Repair of turbine blades of aircraft engines by surfacing. Avtomatich. Svarka, 5, 54-56.

2. Petrik, I.A., Peremilovsky, I.A. (2001) Further development of technology for strengthening of flange platforms of turbine blades from heat-resistant alloys. Tekhnolog. Sistemy, 3, 90-92.

3. Bratukhin, A.G. (2003) Modern aviation materials: Technological and functional peculiarities. Moscow: Aviatekhinform.

4. Kablov, E.N. (2001) Cast blades of gas turbine engines (alloys, technology, coatings). Moscow: MISiS.

5. Boguslaev, V.A., Muravchenko, V.M., Zhemanyuk, P.D. et al. (2003) Technological assurance of service characteristics of parts of gas turbine engine. Blades of turbine. Pt 2. Zaporozhie: Motor Sich.

6. Yushchenko, K.A., Savchenko, V.S., Yarovitsyn, A.V. et al. (2010) Development of the technology for repair microplasma powder cladding of flange platform faces of aircraft engine high-pressure turbine blades. The Paton Welding J., 8, 21-24.

7. (2010) Technological Seminar of Deloro Stellite in Zaporozhie. Ibid., 1, 46-49. 